\title{
Mobility Prediction based Routing in Mobile Adhoc Network using Hidden Markov Model
}

\author{
Rashmi Nagwani \\ M.Tech Scholar \\ Department of C.S.E. \\ Technocrats institute of Technology \\ Bhopal (M.P.)
}

\author{
Deepak Singh Tomar \\ Assistant Professor \\ Department of C.S.E. \\ Technocrats institute of Technology \\ Bhopal (M.P.)
}

\begin{abstract}
Dynamic network topology of Mobile Ad hoc networks (MANETs) which is a consequence of node mobility, make routing a denoting challenge and routes are subject to recurrent disconnections. In such a context, it is important to limit interruptions caused by the node mobility. This paper presents exploiting the concept of routing through predicting the mobility of nodes and therefore reducing the overhead by eliminating transmissions of control packets which are required otherwise for route reconstruction. Also, former awareness of patterns of mobile nodes can be used to suitably allocate resources and upgrade the quality of service (QOS) experienced by mobile devices. This paper proposes Hidden Markov Model (HMM) approach for predicting the mobility of nodes to reduce the overhead head of routing for route discovery.
\end{abstract}

\section{Keywords}

Mobility Prediction, Hidden Markov Model, Routing, MANET, DSR.

\section{INTRODUCTION}

Mobile Ad hoc Network (MANET) [1],[2] consists of a number of mobile nodes that dynamically configures themselves into transient networks topologies without any pre-existing infrastructure. Each node act as host and also as a router as per the requirements to forward packets for those mobile nodes which are not in each other transmission range. Manets are established in emergency situations like natural disasters or military conflicts where routes are typically multihop and network hosts communicate via packet radios. In such environments, it is critical to transmit data packets to destinations efficiently without creating needless overhead. Therefore, the routing protocol used in a MANET is of principal concern because it decides how a data packet is transmitted over multiple hops. A major factor that affects performance of routing protocol is the mobile nature of nodes. An efficient routing protocol is one which adapts to frequently changing topology of the network maintaining the end user transparency. Routing protocols can be categorized into reactive and proactive nature on the basis of how they maintain path through other nodes in the network.

Various Routing protocols have been proposed for MANETs [3], [4], [5], [6]. Some of them being reactive in nature and they do not establish new paths until existing paths break. The recurrent breakage of links requires certain amount of time for repairing existing routes or establishment of new routes. This paper proposes a method to establish route through hops which are more stable. The concept of Hidden Markov Model to predict the future locations of nodes in the network is used here. During route discovery route is selected through those hops whose probability to stay in the transmission range more than others.

\section{OBJECTIVES OF THE WORK}

- To predict the future location(s) of mobile node using Hidden Markov Model (HMM).

- To improve routing by reducing overhead of frequent disconnections due to node mobility.

- To perform comparative analysis of results of the proposed method versus Dynamic Source Routing Protocol.

\section{BACKGROUND}

The assessment of the future position of a node is called its Mobility Prediction. The meaning of 'position' varies with the kind of wireless network being used. For infrastructure base networks, the meaning of position is the access point (AP) to which the mobile node is connected. Mobility prediction is mainly used to set aside aforetime, the serviceable next AP before the mobile node moves from its current AP, in order to reduce the cutoff time between mobile nodes. While in case of Infrastructure less networks Like Mobile Adhoc Networks (MANETs), the position of any mobile node is traced by it geographical co-ordinates.

In the proposed work, it is assumed that each node in the network knows its position i.e., its geographical co-ordinates which they can learn using typically a GPS receiver. They record their positions at periodic intervals. These positions will define node trajectory. On the basis of previous positions observed we will be able to predict its position at any future time instant $\mathrm{T}$. This comes under a particular case of Time Series Prediction. Position of any node N is represented here using its three co-ordinates namely $(\mathrm{X}, \mathrm{Y}, \mathrm{Z})$. Position time series data is the sequence of positions of mobile nodes over a specific period of time. The mobility prediction can be achieved by location computation, which allows us to predict short-term movements. Here, HMM is used in a new way to predict future location of any mobile node given its previous location co-ordinate.

\section{RELATED WORK}

HMMs have been applied to many areas of time series prediction such as speech recognition [11], financial time series analysis [12], malfunctioning of semiconductor [13] etc. Since, Mobility Prediction is also a special case of Time Series Prediction therefore it is used here to model the 
mobility of nodes using Hidden Markov Model (HMM). It is used as a statistical location prediction tool here.

Many prediction schemes proposed in the field of Manet are based on predefined mobility of nodes using an underlying mobility model. For instance in [7], the prediction of location of nodes is based on the assumption that they use random walk mobility model. As a result such prediction schemes strongly imitate the location of mobile nodes but they don't work satisfactorily in case the assumption of underlying mobility model doesn't exist.

In [8], authors used the linear prediction model to predict mobility of nodes which uses the calculations of speed and direction of movement of mobile node. They assume that the mobile nodes in the network moves with constant speed and direction.

The concept of Neural Network [9] and Bayesian Network [10] has also been used to predict the future locations of mobile nodes. [9] uses a neural network to study Network Access Points (NAP) that a mobile user has traversed based on this it predicts its next NAP. Simplification of process of model generation is the key advantage of such algorithms as logical topology of NAP information can be obtained directly from the system.

\section{THE PROPOSED WORK}

The proposed algorithm works in two phases namely Mobility Prediction using HMM and Routing.

\subsection{MOBILITY PREDICTION USING \\ HMM}

It is assumed that each node in the network knows its position i.e., its geographical co-ordinates which they can learn using typically a GPS receiver. They record their positions at periodic intervals. These positions will define their respective node trajectory. Consider a typical Mobile AdHoc Network scenario as shown in Fig 1.

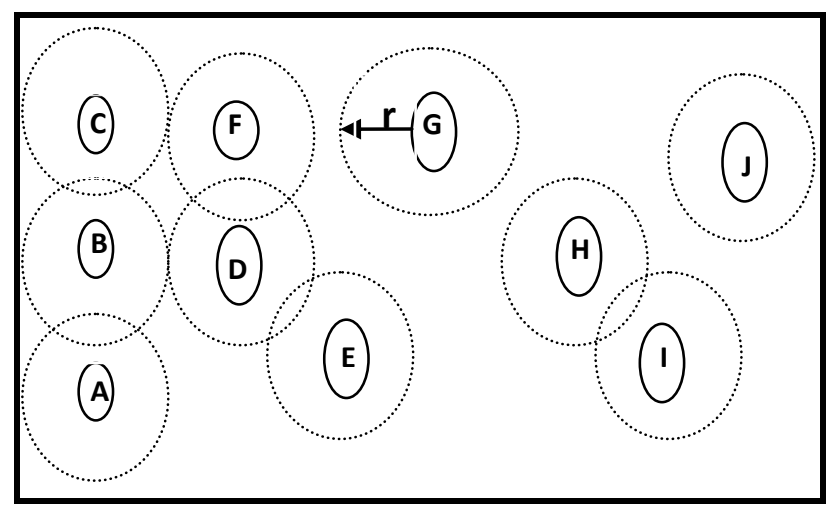

Fig 1: A typical MANET Scenario

Fig 1 consists of 10 nodes. Let ' $r$ 'denote the transmission range of nodes represented through dotted circle. Nodes which come under each other transmission range can send messages to each other. As the nodes are mobile, nodes which were in transmission range of each other will not necessarily stay for a long time. Hence, there is a need of mobility prediction so that it can identify the node with maximum stability and establish route taking that node as our intermediate hop. To predict the mobility of a node the metric of its position vector denoted by ' $\mathrm{P}$ ' is used here. A position or position vector is a Euclidean vector which represents the position of a point in space .Consider a point whose location in space is specified with Cartesian coordinates (e.g., $\mathrm{P}(\mathrm{x}, \mathrm{y}$, $\mathrm{z})$ ). To know whether two nodes come into the transmission range of each other the distance between two points is calculated. Since, each node knows its position in the network from the time when system brought up. At any time instant $t$, let the position of node be $\mathrm{Pt}=(\mathrm{Xt}, \mathrm{Yt}, \mathrm{Zt})$. To predict the position of node at time $t+1$ Hidden Markov Model is used.

A HMM is defined by a finite set of states (some hidden and some observable), a set of state transition probabilities, an alphabet of output symbols (also referred to as emissions) and a set of emission probabilities. The emission probabilities denote the distribution of output symbols that are emitted from each state [11]. A HMM denoted by $\lambda=(\pi, \mathrm{A}, \mathrm{B})$ is well known and widely used statistical source model which models various hidden parameters.

i. $S:\left\{S_{1}, S_{2}, \ldots . S_{N}\right\}$ are the $N$ hidden states in the system. Even though the states are hidden, they have practical significance.

ii. $O:\left\{O_{1}, O_{2}, \ldots . O_{N}\right\}$ are the values of the observed sequences. They symbolize the discrete alphabet size and represent the physical output of the system being modeled.

iii. $\lambda=\{\pi\}$ The initial state probabilities. Here $\pi_{i}$ indicate the probability of starting in state $i$.

iv. $A=\left\{a_{i j}\right\}$ are the state transition probabilities where $a_{i j}$ denotes the probability of moving from state $i$ to $j$. In a system where a state can reach any other state in a single transition, $j>0 \forall i, j$.

$$
a_{i j}=P\left(t_{k}=\mathrm{s}_{j} \mid t_{k-1}=\mathrm{s} i\right)
$$

v. $B=\left\{b_{i k}\right\}$ are the observation state probabilities where $b_{i k}$ is the probability of emitting symbol $k$ at state $i$.

$$
b_{i k}=P\left(o_{k} \mid t_{k}=\mathrm{s}_{j}\right)
$$

1 . The probability of the observation sequence $\mathrm{O}$ is

$$
\begin{aligned}
& P(B \mid S, \lambda)=\prod_{i=1}^{k} P\left(B_{k} \mid S_{k}, \lambda\right) \\
& \quad b_{s 1}(\mathrm{o} 1) . b_{s 2}(\mathrm{o} 2) . b_{s 3}(\mathrm{o} 3) \ldots \ldots \ldots b_{s k}(\mathrm{o} k)
\end{aligned}
$$

2 . The probability of any state sequence is

$P(S \mid \lambda)=\pi_{i 1} S_{i 1 i 2} S_{i 2 i 3 \ldots \ldots \ldots \ldots . . .} S_{a(k-1) a k}$

3. Hence we have,

$\mathrm{P}(\mathrm{B} \mid \lambda)=\sum_{\mathrm{I}} \mathrm{P}(\mathrm{B} \mid \mathrm{S}, \lambda) \cdot \mathrm{P}(\mathrm{S} \mid \lambda)$

If the observed sequence of movements is denoted by $B_{1}, B_{2} \ldots \ldots \ldots . B$.

Lastly the value of $\lambda$ is determined to maximize $\mathrm{P}(\mathrm{B} \mid \lambda)$ i.e., to train the model given a set of observations. This can be done by computing new $\lambda$ based on $\lambda_{0}$ and given $B$. The iteration stops when

$$
\log P(B \mid \lambda)-\log P\left(B \mid \lambda_{B}\right)<\varepsilon
$$

In the proposed work, the objective is to predict the next position for a mobile node using described above HMM model. For training the model, past positions of mobile node were used .The parameters used to train the model were as follows: 
Input: Position vector of Node $\mathrm{N}$ at time instant $\mathrm{t}$.

Output: next Position vector of Node $\mathrm{N}$ at time instant $\mathrm{t}+1$

Once the position of nodes are predicted for successive time intervals the model can choose the most stable hop nodes and thus improves the efficiency of routing. Distance between two nodes $\mathrm{N}_{\mathrm{i}}$ and $\mathrm{N}_{\mathrm{j}}$ having positional co-ordinates $(X i, Y i, Z i)$ and $(X j, Y j, Z j)$ can be calculated using formula given below:

$$
D i j=S Q R T\left((X j-X i)^{2}+(Y j-Y i)^{2}+(Z j-Z i)^{2}\right)
$$

The link will get expired between two nodes when one gets away from the transmission range of other i.e., when the distance between two nodes exceeds the transmission range $r$.

$$
D i j>r
$$

\subsection{ROUTING}

A simple Mobile Ad Hoc network scenario is shown in Fig.1 where each node is represented along with its transmission range ' $r$ '. It consists of ten nodes. Let ' $A$ ' be our source node and $\mathrm{J}$ be the destination node. A want to exchange data with $\mathrm{J}$ but can't do that because ' $\mathrm{J}$ ' is not in its transmission range. So, 'A' would establish a Routing Path to reach up to ' $\mathrm{J}$ '. Nodes which come in range of A's transmission are ' $B$ ' and ' $D$ '. Instead of sending Rreq to both nodes ' $B$ ' and ' $D$ ' , ' $A$ ' will select of node having highest probability of staying in the transmission range for maximum possible time i.e., the most stable hop using HMM mobility prediction as described in section 5.1. Once the stable hop is discovered ' $A$ ' will send Rreq message to that hop and the process continues till the destination node is reached. Upon receiving the Rreq message Node ' $\mathrm{J}$ ' examines its route cache if a route to ' $\mathrm{A}$ ' is found, it will be used as route for sending Rrep. Alternatively, discovery of route to $S$ will be performed.

\section{ROUTING ALGORITHM WITH THE AID OF MOBILITY PREDICTION}

We assume that each node in the network know about its positional co-ordinates which can be obtained through any geopositioning system.

1. Each active node of the network will undergo the prediction method using Hidden Markov Model to know about their successive predicted locations $\mathrm{O} 1, \mathrm{O} 2$, $\mathrm{O} 3$.... .On (for $n$ time intervals ) .

1.1. A HMM $\lambda=(\pi, \mathrm{A}, \mathrm{B})$ can be trained to predict the future location of any mobile node given the system parameters and previous location of node using equation (6) as described in section 5.1.

1.2. When mobile node ' $\mathrm{m}$ ' will get its observation sequence $\mathrm{O}_{\mathrm{m} 1}, \mathrm{O}_{\mathrm{m} 2}, \mathrm{O}_{\mathrm{m} 3} \ldots \ldots \ldots \ldots . . . \mathrm{O}_{\mathrm{mn}}$ so it can provide its predicted locations to other nodes in the network which want to establish path through it.

1.3. As an output of HMM every mobile node will be having their predicted observation sequence.

2. Initially, Source nodes which want to establish a routing path with the destination node will send a message to those neighbor nodes which are in its transmission range asking for their observation sequence.
3. Source node will send Rreq message to those nodes which are supposed to be in its transmission range for maximum possible time than others.

4. As a result, source node will find its stable intermediate hop.

5. Similarly, other nodes will find their stable hops by looking at the observation sequence of their radio range neighbors, until they reach the destination node or a node having the path to the destination.

6.1. If the node is some intermediate node having stable path to the destination node than it concatenates its source to destination route to the aggregated route in the Rreq, and stores this new route in the Rrep.

6.2. Otherwise, if the node is the destination node itself, then it answers by placing the aggregated route from Rrep to Rreq.

Since, the nodes selected as intermediate hop are the stable one therefore the need to maintain the route discovered will be reduced to a significant extent.

\section{PERFORMANCE EVALUATION}

In this section, the performance of our algorithm via simulations is estimated. The modeling and evaluation of the proposed algorithm were realized in the OPNET Modeler simulation environment [14] which is a well-known software tool for simulation and analysis of different type of network technologies, architectures and protocols. The nodes were randomly placed in a $1000 \times 1000 \mathrm{~m}$ campus environment. The mobile network consists of random number of mobile nodes with each node having constant speed and random mobility. The number of nodes is varied as multiples of 10 as $10,20 \ldots 40$. The transmission range of node can be specified with the help of it's transmit power. The parameters that have been used in the following experiments are summarized in Table I.

Table I: Simulation Parameter

\begin{tabular}{|c|c|}
\hline Simulation Parameters & Values \\
\hline Simulator & OPNET 14.0 \\
\hline Simulation Area (m) & $1000 \times 1000$ \\
\hline Network Size & $10,20,30,40$ Nodes \\
\hline Simulation Time(sec) & 3600 \\
\hline Data rate(bps) & $11 \mathrm{Mbps}$ \\
\hline Packet Inter-Arrival & exponential (1) \\
\hline Time (s) & exponential (1024) \\
\hline Packet size (bits)
\end{tabular}

The results in two different scenarios are analyzed and described below:

Scenario 1: Comparison of results of routing with Dynamic Source Routing Protocol and HMM Mobility Prediction method 


\subsection{Number of Hops per Route versus Simulation Time}

In terms of Number of Hops required per route between our proposed algorithm and DSR, our proposed algorithm works better than DSR as Hops reduced with an average of $30 \%$ as shown in Fig. 2.

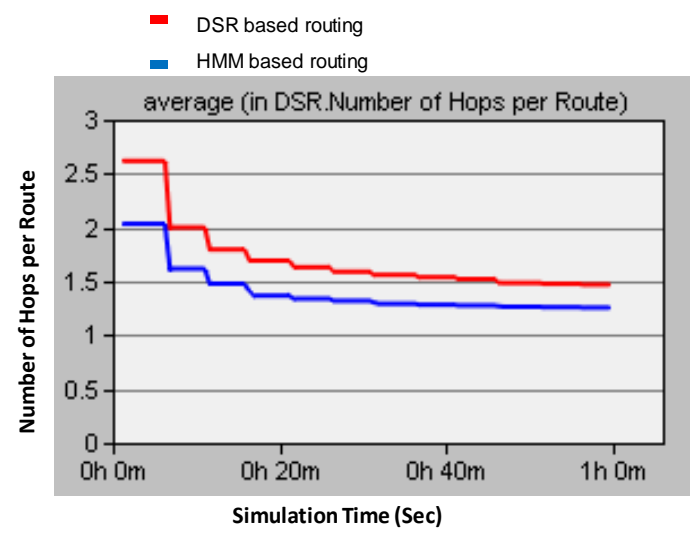

Fig 2: Results shows that number of hops required by proposed method is lesser than DSR.

\subsection{Route Discovery Time versus Simulation Time}

The time to discover a route to a specific destination is the time when a route request Rreq was sent out to discover a route to that destination until the time a route reply Rrep is received with a route to that destination. Fig. 3 shows that routing when performed with HMM Mobility Prediction method results much reduced Route Discovery time than DSR protocol.

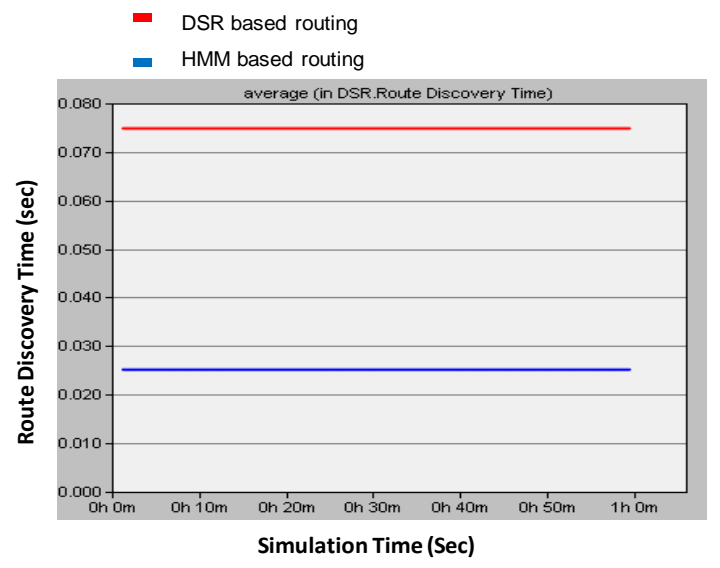

Fig 3: Route Discovery Time obtained is much lesser than DSR

\subsection{End To End Delay versus Simulation Time:}

End-to-end delay refers to the time taken for a packet to be transmitted across a network from source to destination. As shown in Fig. 4 End to End Delay is much lesser than the delay caused by DSR protocol because route maintenance is excessively required in DSR case.

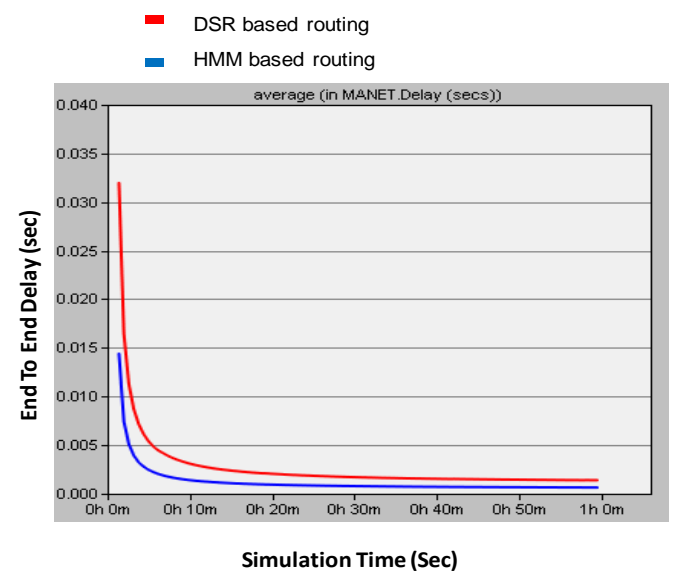

Fig 4: End To End Delay obtained by DSR protocol is greater than the proposed method

\subsection{Retransmission Attempts versus Simulation Time}

Figure 5 shows that the numbers of retransmissions required by DSR protocol are much higher than our proposed algorithm before it can successfully transmit data.

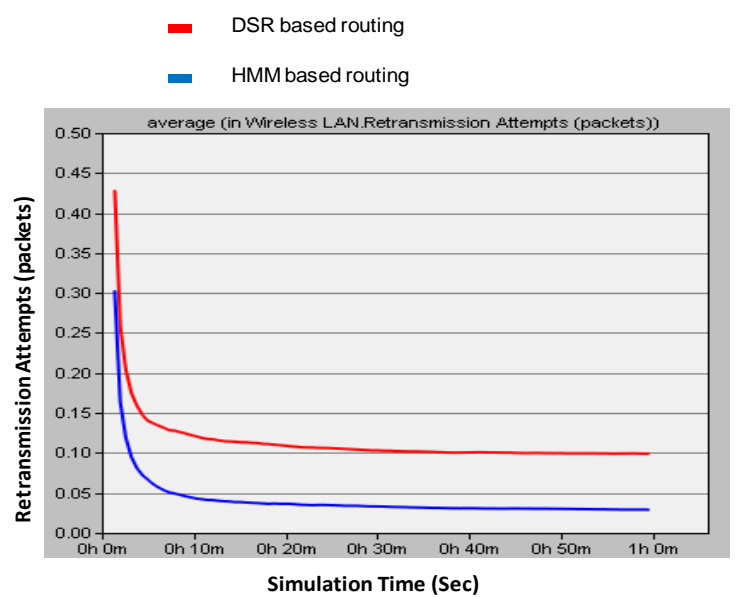

Fig 5: Retransmission attempts are also lesser than the DSR Protocol

\subsection{Throughput versus Simulation Time}

It is defined as the ratio of the total data reaches a receiver from the sender. It is the average rate of successful message delivery over a communication channel. The throughput is usually measured in bits per second (bit/s or bps).

The result of throughput obtained by performing routing with DSR protocol and HMM based Mobility prediction method is shown in Fig. 6. When simulation was performed with 20 nodes, throughput was less but as the number of nodes 
increased in the network it improved significantly as shown in Fig 11.

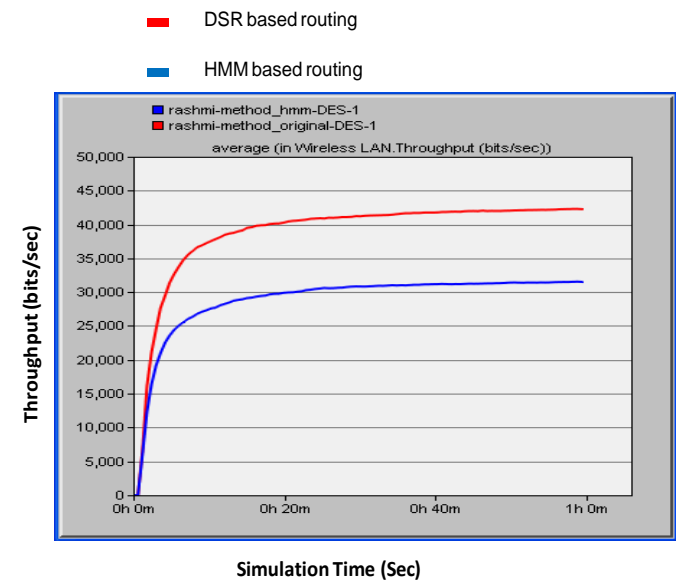

Fig 6: Throughput result of the proposed method as compared with DSR protocol

Scenario 2: In the second scenario we varied the number of nodes from 10 to 40 . The results obtained of routing when performed with different network size are shown below.

\subsection{Number of Hops per Route versus Simulation Time}

In case of 10 nodes, No. of Hops per route are constant but decreases linearly as we increase network size.

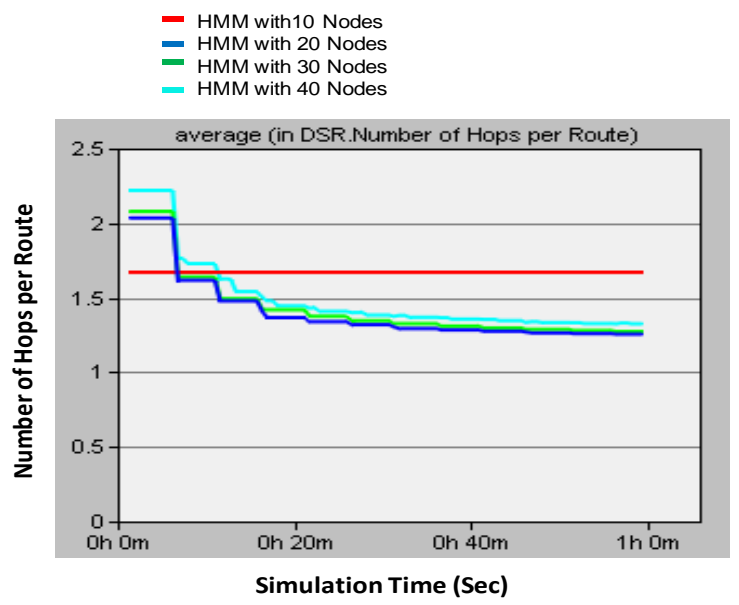

Fig 7: No. of Hops per Route varies linearly with network size but is constant for network size $=10$ nodes

\subsection{Route Discovery Time versus simulation Time}

Fig 8 shows that for initial simulation time of $400 \mathrm{Sec}$, Route Discovery Time is maximum for network size of 40 nodes but later on it stabilizes to a constant value of $0.06 \mathrm{sec}$. For network of 30 nodes the value of Route Discovery time 0.07 sec. Whereas, for network of 20 nodes Route Discovery is accomplished in $0.02515 \mathrm{sec}$ and for 10 nodes its $0.01256 \mathrm{sec}$.

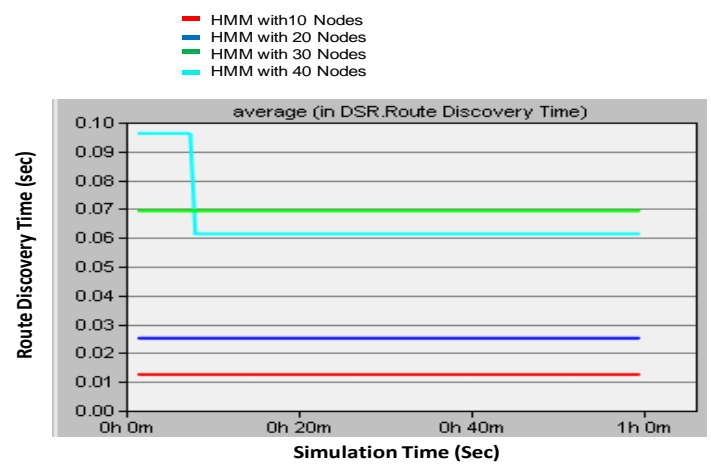

Fig 8: Results of Route Discovery Time for variation of network size from 10 to 40 nodes

\subsection{End To End Delay versus Simulation Time}

End to End Delay varies proportional to the size of network.

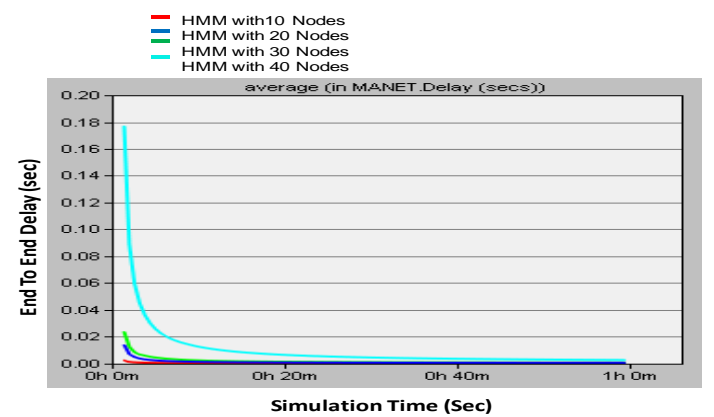

Fig 9: Results of End to End Delay for variation of network size from 10 to 40 nodes

\subsection{Retransmission Attempts \\ versus Simulation Time}

There is direct proportion between the size of network and the number of retransmission attempts. The results of retransmission attempts are shown in Fig 10.

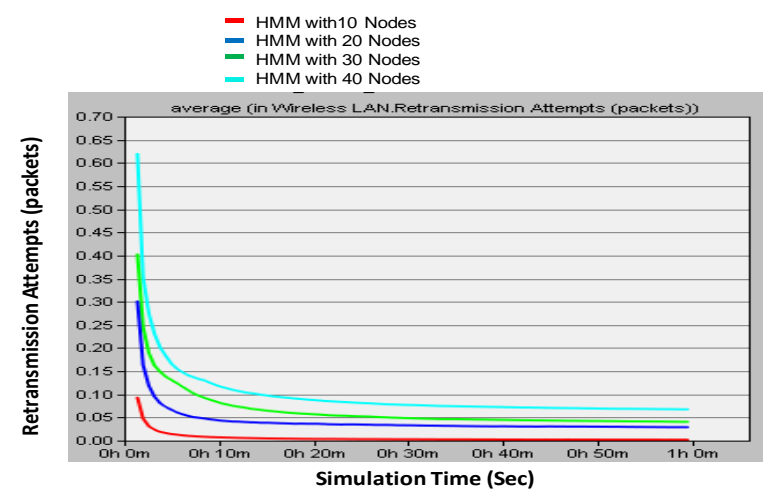

Fig 10: Retransmission attempts 


\subsection{Throughput versus Simulation Time}

Throughput is analyzed here by varying the network size from 10 nodes to 40 nodes. As we increased the network size throughput also increases gradually as shown in Fig 11.

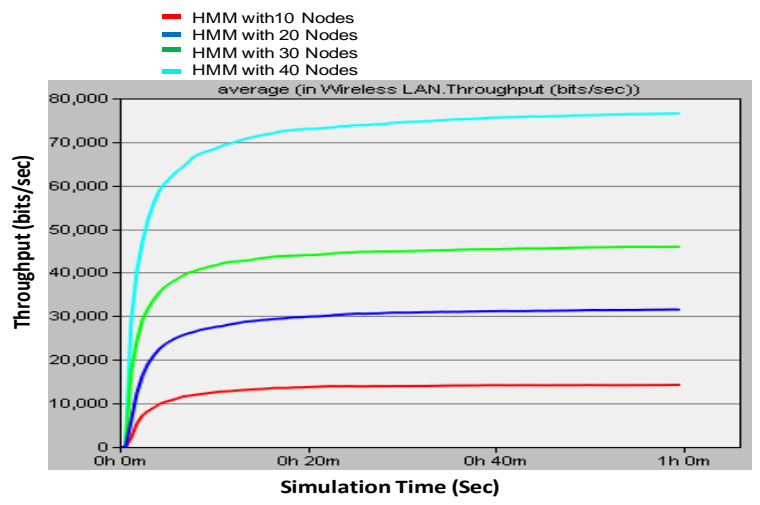

Fig 11: Results of Throughput (bits/sec) for variation of network size from 10 to 40 nodes

\section{CONCLUSIONS}

Routing of Manet is improved by the use of Mobility Prediction of nodes in the network which helps in selecting stable intermediate hops and also helps in forecasting their location. In this study the concept of Hidden Markov Model for time series prediction is used. The results of the proposed work are compared with the well known Dynamic Source Routing Protocols.

As a result, of the proposed work it is found that the route discovery time of our protocol is much lesser than the DSR protocol. Number of Hops, End to End Delay and the Retransmission attempts has also been reduced. Whereas throughput obtained was less initially when compared with the DSR protocol. But it improved as the size of the network is increased.

\section{FUTURE WORK}

In this paper, the use of Hidden Markov Model in improving the routing procedure in Mobile Ad hoc Network is shown. In future the mobility prediction can be used as a metric of forming Clusters in the network. As clustered network are getting much attention these days and various techniques are getting evolved to form most stable clusters. So, mobility prediction with the use of HMM could be used to form stable clusters. In addition to that the system can also be enhanced by using other intelligent techniques for mobility prediction like Ant Colony Optimization.

\section{REFERENCES}

[1] Magnus F., Per Johansson, and Peter L. "Wireless ad hoc networking: the art of networking without a network," Ericsson Review, No.4, 2000, pp. 248-263

[2] IETF Working Group: Mobile Adhoc Networks (manet).http://www.ietf.org/html.charters/manetcharter.html.

[3] V. D. Park and M. S. Corson. "Temporally-ordered routing algorithm (TORA) Version 1, functional specification". IETF, Internet Draft, draft-ietf-manettora-spec-02.txt, October 1999.

[4] David B. J; David A. M.; Josh B. (1999). DSR: The dynamic source routing protocol for multi-hop wireless Ad Hoc networks.

[5] Charles. E. P., Elizabeth M. R., and Samir R. D. "Ad Hoc on-demand distance vector (AODV) routing". IETF internet draft (work in progress), Internet Engineering Task Force, November 2002.

[6] Young B. K. and Nitin H. V., "Location-aided routing (LAR) in mobile Ad Hoc networks". In Proceedings of ACM/IEEE MOBICOM' 98, Dallas, Texas, Oct 1998, pp $66-75$

[7] A. B. McDonald and Taleb. F. Z., IDesign and Simulation of a Distributed Dynamic Clustering Algorithm for Multimode Routing in Wireless Ad Hoc Networks," SIMULATION, 2002, vol. 78, no. 7, pp 408-422.

[8] Sung-Ju L., William S., and Mario G. Mobility prediction in wireless networks. In Proceedings of IEEE ICCCN, October 2000, pages 22.25.

[9] Joe C., Raouf B., "Mobility Prediction in Wireless Networks using Neural Networks". MMNS 2004: 320334.

[10] Petri K., Petri M., Teemu. R., Henry T., Kimmo V. and Hannes W. Probabilistic Methods for Location Estimation in Wireless Networks. In Emerging Location Aware Broadband Wireless Ad Hoc Networks, Springer US, 2005, pages 173-188.

[11] L. Rabiner, 'A tutorial on hidden markov models and selected applications in speech recognition', Proceedings of the IEEE, Feb 1989vol. 77, no. 2, pp. 257-286.

[12] Yingjian Z. Prediction of financial time series with Hidden Markov Models. Master's thesis, The School of Computing Science, Simon Frazer University, Canada, 2004.

[13] Xianping G. and Padhraic S. Deformable Markov model templates for time-series pattern matching. In Proceedings of the sixth ACM SIGKDD international conference on Knowledge discovery and data mining, pages 81-90. ACM Press New York, NY, USA, 2000.

[14] OPNET TECHNOLOGIES, INC. (2005, 25 Septiembre). OPNET: Making networks and applications perform. Bethesda (USA): OPNET Technologies, Inc. http://www.opnet.com 